\title{
A U.S. Cost Analysis of Triplet Regimens for Patients with Previously Treated Multiple Myeloma
}

\author{
Sarah Hollmann, MBiotech; Daniel Moldaver, PhD; Nik Goyert, MA; Daniel Grima, MSc;
} and Eric M. Maiese, MHS, PhD

\begin{abstract}
BACKGROUND: In recent years, the FDA has approved several 3-agent (i.e., triplet) combinations for previously treated multiple myeloma (MM), and the National Comprehensive Cancer Network (NCCN) now recommends triplet regimens over doublets. Little is known about the real-world cost of triplet combinations because of the limited time that they have been on the market since FDA approval. Furthermore, traditional cost analyses developed to support market entrance rely on utilization assumptions that are difficult to validate when numerous comparators simultaneously enter the market.
\end{abstract}

OBJECTIVE: To perform a 1-year cost analysis of novel triplets used for the treatment of patients with previously treated MM controlling for differences in utilization.

METHODS: FDA-approved, NCCN-recommended (preferred and category 1 for previously treated MM) treatments included in the analysis were daratumumab plus lenalidomide plus dexamethasone (DARA/LEN/DEX), daratumumab plus bortezomib plus dexamethasone (DARA/BOR/DEX), elotuzumab plus lenalidomide plus dexamethasone (ELO/LEN/DEX), carfilzomib plus lenalidomide plus dexamethasone (CAR/LEN/DEX), and ixazomib plus lenalidomide plus dexamethasone (IXA/LEN/DEX). To control for market uptake, the model was designed to estimate the cost of treating an average patient over a 1-year time horizon. Drug administration and dosing, required comedications, postprogression therapy, monitoring requirements, and adverse event (AE) rates were based on FDA prescribing information or clinical trials. $A E s \geq$ grade 3 that occurred in $\geq 5 \%$ of patients were included. RED B00K wholesale acquisition costs were used for drug acquisition costs. Costs of drug administration, AE management, and patient monitoring were based on the 2018 Center for Medicare \& Medicaid Services payment rates or from published literature (inflated to 2018 U.S. dollars). The treatment duration for each regimen was estimated from modeled progression-free survival data; the 12-month progression-free survival rate was assumed to be equivalent to the probability that an average patient remained on therapy for at least 1 year after treatment initiation, which was used to estimate time-depended treatment-related costs. The probability of progression within 1 year of treatment initiation was used to inform the average postprogression therapy costs for each regimen.

RESULTS: The estimated cost per patient for each triplet regimen was \$13,890 (DARA/BOR/DEX), \$22,231 (IXA/LEN/DEX), \$24,322 (ELO/LEN/ DEX), $\$ 26,410$ (DARA/LEN/DEX), and \$27,432 (CAR/LEN/DEX). Drug acquisition costs and treatment duration were the largest drivers of cost. Scenario analyses with plausible alternative input parameters found the maximum per month cost of therapy to be $\$ 30,657$ (CAR/LEN/DEX) and the minimum per month cost of therapy to be $\$ 13,784$ (DARA/BOR/DEX).

CONCLUSIONS: This analysis controlled for differential utilization rates for 5 FDA-approved, NCCN-recommended triplet therapies for the treatment of previously treated MM. Of the examined regimens, treatment with DARA/ BOR/DEX was estimated to have the lowest average monthly cost per patient, while CAR/LEN/DEX was the most expensive. As is common with modeling, some assumptions were necessary, and results may not be generalizable.

J Manag Care Spec Pharm. 2019;25(4):449-59

Copyright $\odot 2019$, Academy of Managed Care Pharmacy. All rights reserved.

\section{What is already known about this subject}

In the United States, multiple myeloma is one of the most common hematological malignancies, and it is estimated to account for 12,770 deaths in 2018 .

Since 2012, a number of new therapies (e.g., daratumumab, elotuzumab, carfilzomib, and ixazomib) have received FDA approval and are now recommended by the National Comprehensive Cancer Network and are preferred treatment for previously treated multiple myeloma patients, when used in triplet combinations.

As standard cost analyses (e.g., budget impact models) developed to support market entrance rely on utilization/uptake rate assumptions, little is known about the real-world costs of triplet therapies that have recently received FDA approval.

\section{What this study adds}

This cost analysis estimated the direct costs to a U.S. health insurance plan, during the first year of treatment of patients with previously treated multiple myeloma with 5 common triplet therapies (daratumumab, carfilzomib, elotuzumab, and ixazomib in combination with dexamethasone and lenalidomide or bortezomib).

Results from the modeled cost analysis indicate that, over a 1-year time horizon after initiating treatment, daratumumab in combination with bortezomib and dexamethasone is, on average, the least expensive triplet therapy, while therapy with carfilzomib plus lenalidomide and dexamethasone is the most expensive triplet therapy for patients with relapsed or refractory multiple myeloma patients.

One-way sensitivity and scenario analyses were performed and showed that trends in the costs of each regimen remained consistent when alternative assumptions were used.

$\mathrm{M}$ ultiple myeloma (MM) is a clonal malignancy of plasma cells that is one of the most commonly diagnosed hematological cancers in the United States. ${ }^{1}$ The U.S. Surveillance, Epidemiology, and End Results (SEER) program estimated there to be 30,770 new diagnoses of MM and 12,770 MM-related deaths in 2018. ${ }^{2} \mathrm{MM}$ is an incurable disease with limited effective treatment options for relapsed or refractory patients (i.e., previously treated MM). ${ }^{3}$ Historically, 
therapies for MM have included immunomodulatory drugs (i.e., lenalidomide [LEN] and thalidomide), proteasome inhibitors (i.e., bortezomib [BOR]), and use of stem cell transplantation.

However, since 2012, new proteasome inhibitors and immunomodulatory agents, such as carfilzomib (CAR), pomalidomide, panobinostat, and ixazomib (IXA), and monoclonal antibodies, such as elotuzumab (ELO) and daratumumab (DARA), have received approval by the U.S. Food and Drug Administration (FDA) for treatment of relapsed or refractory MM. Use of these novel therapies in combinations with dexamethasone (DEX) and BOR or LEN as triplet therapies represents a promising approach for relapsed or refractory MM with some combinations, such as DARA in combination with LEN and DEX, achieving 12-month progression-free survival rates (PFS) rates above 80\%., Indeed, the triplet regimens DARA/LEN/DEX, DARA/BOR/DEX, ELO/ LEN/DEX, IXA/LEN/DEX, and CAR/LEN/DEX have received a preferred (category 1) recommendation from the National Comprehensive Cancer Network (NCCN) for patients with previously treated MM. ${ }^{6} \mathrm{NCCN}$ also broadly recommends using triplet regimens over doublets. ${ }^{6}$

In contrast to the understanding of the efficacy of triplet therapy for patients with relapsed or refractory MM, little is known about the real-world cost of these triplets due to limited time on the market since FDA approval. Real-world utilization data of these newer triplets remain immature, and few studies have been found that estimate the cost of triplet therapies-most that exist occurred before the FDA approval of numerous triplet therapies. ${ }^{7-9}$ In light of rising health care costs as a proportion of the gross domestic product in the United States ${ }^{10}$ key decision makers, such as pharmacy benefit managers (PBMs) and even professional organizations such as the American Society of Clinical Oncology (ASCO), are increasingly turning to value-based assessments of medicines in an effort to evaluate therapies in a systematic fashion to optimize patient outcomes while balancing budgetary requirements. ${ }^{11}$

This cost analysis sought to estimate the average costs of treating relapsed or refractory MM patients with triplet combinations, during the first year of treatment. Triplets analyzed (DARA/ LEN/DEX, DARA/BOR/DEX, ELO/LEN/DEX, CAR/LEN/DEX, and IXA/LEN/DEX) were FDA approved and NCCN recommended (preferred, category 1) for previously treated MM. ${ }^{6}$ To account for differences in utilization that might have confounded the results, the modeled analysis was designed to estimate the average cost of treating 1 patient with each regimen.

\section{Methods}

\section{Model Structure}

The model was developed to estimate the 1-year direct costs of 5 triplet regimens used for the treatment of patients with previously treated MM; this model was developed by removing market utilization estimates from an existing budget impact model. ${ }^{12}$ The cost analysis was performed from the U.S. payer perspective, over a 1-year time horizon.

Modeling methods used were consistent with best practices of the International Society for Pharmacoeconomic and Outcomes Research (ISPOR) Task Force on Good Research Practices. ${ }^{13}$ No patient data were used or analyzed in this study; therefore, ethics review by an institutional review board was not conducted. Direct costs considered within the model included the cost of drug acquisition, comedication, administration, patient monitoring, adverse event (AE) management, and subsequent therapy in the proportion of patients expected to progress within the time horizon. Data are presented as rounded numbers, but model calculations were performed without rounding.

\section{Model Inputs and Assumptions}

Clinical Inputs and Costs. Triplets considered in the analysis were FDA approved and NCCN recommended (preferred, category 1) for previously treated $\mathrm{MM}^{6}$; these therapies were DARA/ LEN/DEX, DARA/BOR/DEX, ELO/LEN/DEX, CAR/LEN/DEX, and IXA/LEN/DEX. Drug administration time, dosing, monitoring, and required comedications were based on the approved FDA prescribing information for each agent. ${ }^{14-17}$ The cycle length and core regimen dosing in combination with the drug acquisition costs per cycle and 1-year costs of administration, monitoring, and comedications are shown in Table 1. The analysis was designed to assess the average monthly and total cost of the first year after initiating therapy with each regimen on a per-patient basis. Therefore, given that this analysis was meant to reflect a practical approach to costing and considered therapies on a one-to-one basis, market shares were not needed and therefore did not confound results.

At the time of model construction, the average selling price (ASP) was not available for all treatment comparators within the analysis; thus, RED BOOK (May 2018) wholesale acquisition costs (WAC) were used. ${ }^{18}$ For calculation of drug acquisition costs, body surface area was assumed equivalent to the median body surface area found in a retrospective analysis of 1,650 patients who received anticancer drugs $\left(1.86 \mathrm{~m}^{2}\right)$, and weight was assumed equivalent to the median weight of MM patients after high-dose chemotherapy and stem cell transplantation, as reported by a retrospective analysis of $318 \mathrm{MM}$ patients $(79.4 \mathrm{~kg})$. $^{19,20}$

Routine monitoring and laboratory tests recommended within the FDA prescribing information of each regimen were incorporated in the analysis, in addition to regular (once per cycle) physician visits. Costs were derived from the nonfacility national payment amounts reported by the Center for Medicare \& Medicaid Services. ${ }^{21,22}$ Comedications required by the FDA prescribing information were also included, using WAC unit costs. Comedications, testing, and monitoring for specific populations were not considered. 


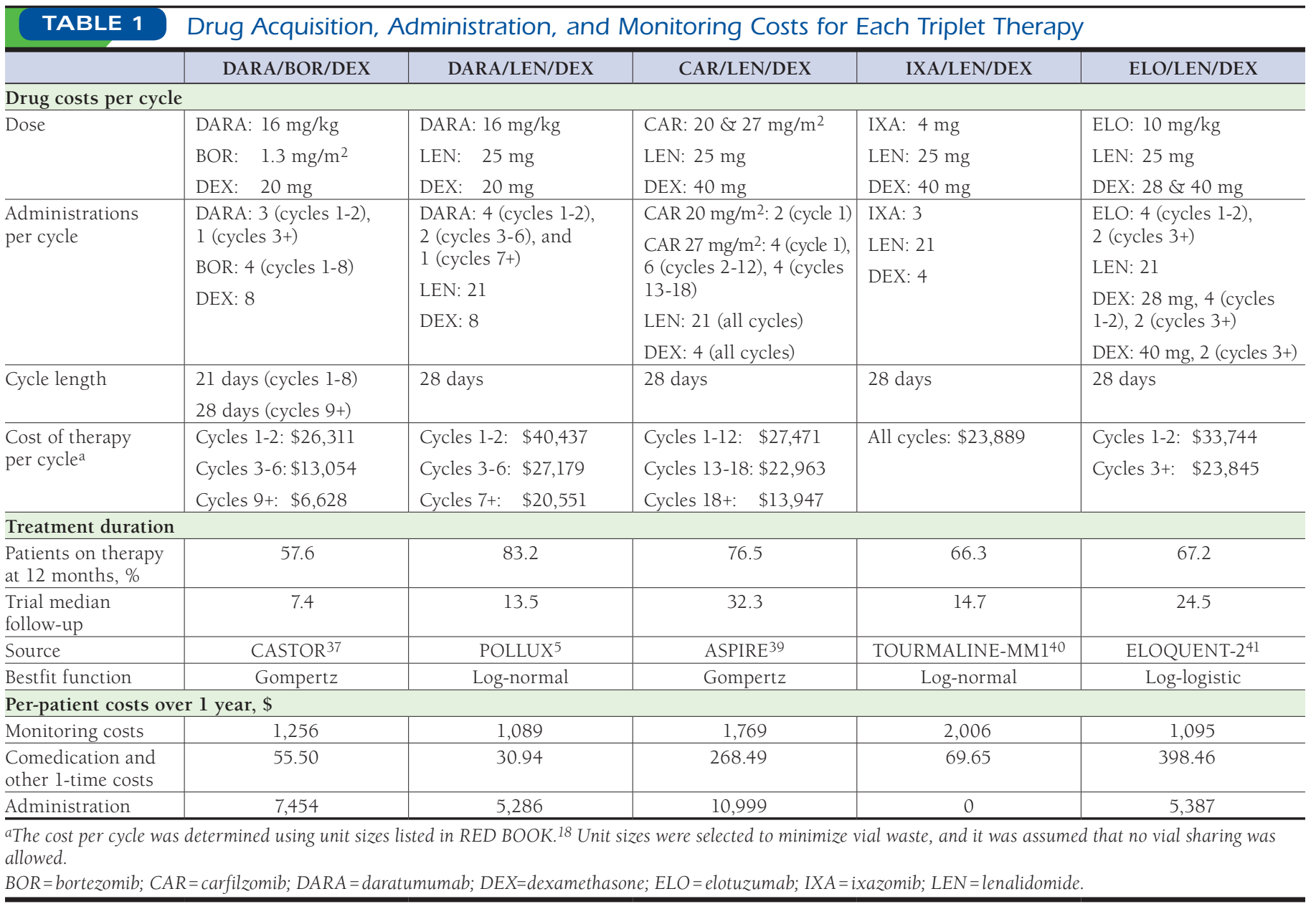

The cost of administering each triplet regimen was based on the 2018 Physician Fee Schedule. ${ }^{22}$ Nonfacility national payment amounts were used within the model; these costs were (a) first hour of intravenous (IV) infusion ( $\$ 144.72$; Healthcare Common Procedure Coding System [HCPCS] code 96413); (b) subsequent hours of IV infusion (\$31.68; HCPCS code 96415); and (c) subcutaneous infusions (\$81.72; HCPCS code 96401). The cost of drug administration was applied to the duration of infusion, as reported within the FDA prescribing information of each regimen. The median duration of infusion for DARA and ELO varied by infusion, with the duration of the first, second, and subsequent infusions of DARA being 7.0, 4.3, and 3.5 hours and 4,3 , and 2 hours for ELO. ${ }^{14,15}$ CAR administration time was estimated to be 10 minutes per IV infusion. ${ }^{16}$ The cost of 1 subcutaneous administration was assumed for each BOR administration, and no cost was inputted for medications administered orally (e.g., IXA and LEN). ${ }^{23}$

The average time-on-therapy for a patient receiving triplet therapy was estimated from PFS Kaplan-Meier curves from the pivotal clinical trial of each triplet therapy. Trial data were digitized using DigitizeIt software version 2.2.2 (Bormisoft, Braunschweig, Germany) and fit with 5 parametric functions (Weibull, exponential, log-normal, log-logistic, and Gompertz). The best fit function was determined through the Akaike and Bayesian information criterion and visual inspection for clinical plausibility. The half-cycle corrected 12-month PFS rate was calculated from the curve of best fit and was used to guide the application of treatment-related costs and subsequent therapy costs. Subsequent MM-related therapy costs were based on the treatment mix and duration reported by Ollendorf et al. (2016) and 2018 RED BOOK WAC pricing. ${ }^{18,24,25}$

Toxicity Costs. The cost of managing grade 3 and above AEs that occurred in $\geq 5 \%$ of patients were considered within the analysis. AE rates for each triplet were derived from the FDA prescribing information (Table 2). The costs of AE management were taken from a recent cost analysis by Roy et al. (2015), which examined the burden of relapsed and refractory MM in 


\section{TABLE 2 Adverse Event Rates and Costs for Each Triplet}

\begin{tabular}{|c|c|c|c|c|c|c|}
\hline $\begin{array}{l}\text { Adverse Events } \\
\text { (Grade } \geq 3 \text { Occurring in } \geq 5 \% \text { of Patients) }\end{array}$ & $\begin{array}{l}\text { Cost } \\
\text { (2018 USD), \$a }\end{array}$ & $\begin{array}{c}\text { DARA/BOR/DEX } \\
\%\end{array}$ & $\begin{array}{c}\text { DARA/LEN/DEX } \\
\%\end{array}$ & $\begin{array}{l}\text { CAR/LEN/DEX } \\
\%\end{array}$ & $\begin{array}{l}\text { IXA/LEN/DEX } \\
\%\end{array}$ & $\begin{array}{c}\text { ELO/LEN/DEX } \\
\%\end{array}$ \\
\hline Neutropenia & 179 & \begin{tabular}{l|l}
15 \\
\end{tabular} & 53 & 27 & 26 & - \\
\hline Anemia & 1,049 & 13 & 13 & 14 & - & - \\
\hline Thrombocytopenia & 179 & 47 & 13 & 15 & 26 & 19.2 \\
\hline Lymphopenia & 179 & 48 & 52 & - & - & 8.8 \\
\hline Leukopenia & 179 & - & - & - & - & 32.4 \\
\hline Pneumonia & 16,049 & - & - & 9 & - & 14.2 \\
\hline Pulmonary/upper respiratory infection & 5,639 & 6 & 7 & - & - & - \\
\hline Hyperglycemia & 179 & - & - & 5 & - & 17.0 \\
\hline Hypokalemia & 1,844 & - & - & 6 & - & - \\
\hline Diarrhea & 10,520 & - & 5 & - & 6 & 5.0 \\
\hline Infusion reaction & $13,301^{\mathrm{b}}$ & 9 & 5 & - & - & - \\
\hline Fatigue & 9,115 & - & 7 & 5 & - & 12.6 \\
\hline Cataracts & $782^{\mathrm{c}}$ & - & - & - & - & 6.3 \\
\hline Hypocalcaemia & 1,248 & - & - & - & - & 11.3 \\
\hline Hyperkalemia & $1,844^{d}$ & - & - & - & - & 6.6 \\
\hline Peripheral neuropathy & 846 & 5 & - & - & - & - \\
\hline \multicolumn{2}{|l|}{ Total cost } & $\$ 1,911$ & $\$ 2,572$ & $\$ 2,242$ & $\$ 724$ & $\$ 4,404$ \\
\hline \multicolumn{7}{|c|}{$\begin{array}{l}\text { aUnless otherwise indicated, adverse event costs were derived from Roy et al. (2015). }{ }^{26} \text { All costs were inflated to } 2018 \text { values. } \\
\text { bThe cost of infusion reactions was determined using a weighted average of the cost of patients requiring inpatient/hospitalization care versus outpatient care as reported by } \\
\text { Foley et al. (2010). }{ }^{27} \\
\text { cAverage cost of national average physician fee for HCPCS cataract removal billing codes (66830-66984). }{ }^{22} \\
\text { dAssumed equivalent to the cost of managing hypokalemia, reported by Roy et al. }(2015) .26 \\
\text { BOR= bortezomib; CAR = carfilzomib; DARA = daratumumab; DEX=dexamethasone; ELO=elotuzumab; HCPCS=Healthcare Common Procedure Coding System; } \\
\text { IXA=ixazomib; LEN =lenalidomide; USD=U.S. dollars. }\end{array}$} \\
\hline
\end{tabular}

the United States when possible. ${ }^{26}$ The cost of managing infusion reactions, cataracts, and hyperkalemia were not reported by Roy et al. ${ }^{26}$ Therefore, although the rate of infusion reactions were based on the reported frequency of each triplet, the unit cost of managing an infusion reaction was assumed to be equivalent for all triplets and was derived from a published retrospective claims-based economic assessment of the cost of infusion reactions that were performed in colorectal cancer patients (i.e., the unit cost of infusion reaction treatment was assumed to be the weighted average cost of treating inpatients and outpatients; alternative assumptions were tested in sensitivity analyses). ${ }^{27}$ The cost of managing cataracts was assumed equivalent to the national average physician fee for HCPCS cataract removal billing codes (66830-66984). ${ }^{22}$ Finally, the cost of managing hyperkalemia was assumed to be equivalent to the cost of managing hypokalemia, as reported by Roy et al. because the cost of recommended treatment for patients with hyperkalemia and hypokalemia is similar. ${ }^{26,28,29}$ Reported costs were inflated to 2018 U.S. dollars using the health component of the Consumer Price Index.

\section{Model Outputs and Analyses}

The economic model estimated the average monthly and annual cost of treating 1 relapsed or refractory MM patient within
12 months of initiating therapy. The average per-patient cost of triplet therapy was determined by multiplying the probability that a patient would remain on therapy at the end of 1 year after initiating therapy, as estimated from PFS Kaplan-Meier data, by the 1-year costs of drug acquisition, administration, monitoring, and comedications. The cost of AE management was applied as a l-time cost for each triplet. The probability that a patient would have progressed within 1 year of treatment initiation (determined as 1 minus the PFS probability at 1 year) was multiplied by the cost of 1 year of subsequent therapy. Analyses for each triplet regimen were performed individually, and all costs are presented in 2018 U.S. dollars.

The sensitivity of the model was assessed through a combination of one-way and scenario analyses. One-way sensitivity analyses were used to identify the most significant model parameters and were performed by varying individual parameters by a set high and low range $( \pm 20 \%)$. Scenario analyses then tested the most significant model parameters with plausible alternative values. Scenario analyses performed included (a) use of available ASP rather than WAC for core regimen unit costs; (b) alternative treatment durations; (c) alternative average patient body surface area/weight; and (d) alternative infusion reaction management costs. To capture the relative effect of parameters on all triplets equally, the one-way 


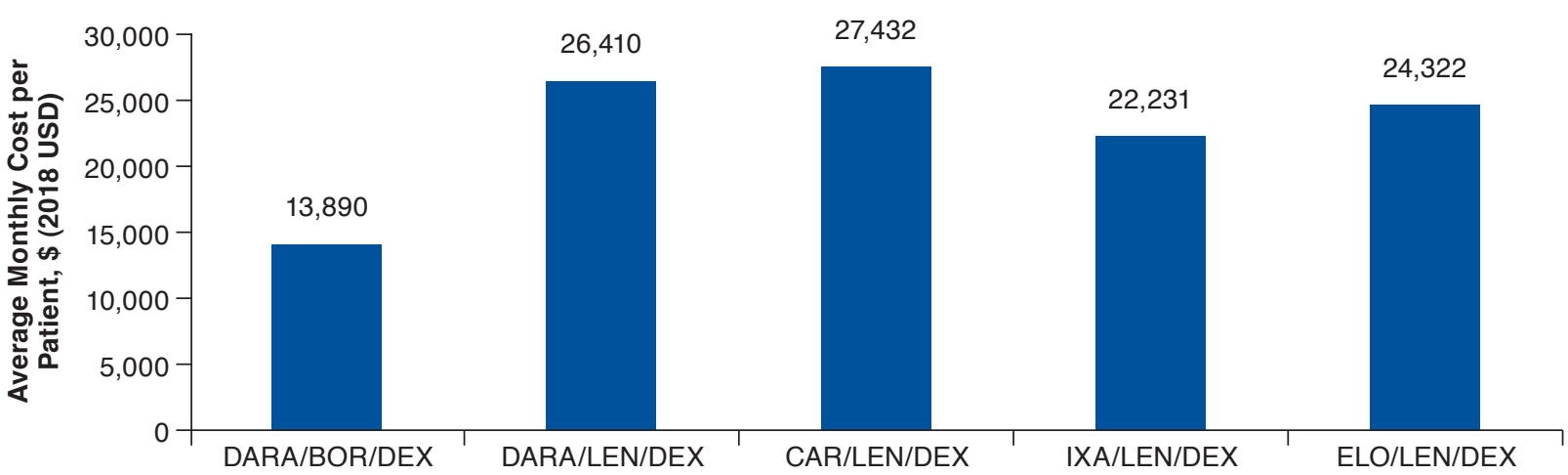

BOR= bortezomib; $C A R=$ carfilzomib; DARA = daratumumab; DEX=dexamethasone; $E L O=$ elotuzumab; IXA =ixazomib; LEN=lenalidomide; USD =U.S. dollars.

sensitivity analysis captured changes in the cumulative average monthly cost of triplet therapy (i.e., the sum of the average monthly cost of DARA/LEN/DEX, DARA/BOR/DEX, CAR/ LEN/DEX, ELO/LEN/DEX, and IXA/LEN/DEX); the output of the one-way sensitivity analysis was the percent change in cumulative per month costs. Scenario analyses were then performed examining individual triplet regimens, with the output being changes in the predicted monthly cost.

\section{Results}

\section{Base Case}

In the base case analysis, the least expensive triplet regimen was DARA/BOR/DEX, with an estimated average monthly cost of $\$ 13,890$ per patient. In contrast, the most expensive triplet regimen was calculated to be CAR/LEN/DEX, with an average monthly per patient cost of $\$ 27,432$. The estimated average monthly cost per patient for each triplet regimen is presented in Figure 1. The estimated cost of treating an average patient for 1 year with each regimen (Table 3) ranged between $\$ 166,676$ (DARA/BOR/DEX) to $\$ 329,178$ (CAR/LEN/DEX). The contribution of the costs of drug acquisition, administration, monitoring, comedications, AE management, other 1-time costs, and subsequent therapy to the annual estimated costs are presented in Table 3. For all triplet regimens, drug acquisition costs were estimated to account for the greatest proportion of the annual cost of treatment. The impact of other cost components was more heterogenous, with the second largest driver of cost being subsequent therapy costs for DARA/BOR/DEX, ELO/LEN/DEX, and IXA/LEN/DEX and administration costs for DARA/LEN/DEX and CAR/LEN/DEX.

\section{Sensitivity Analysis}

Results of the one-way sensitivity analysis indicate that the model, and all 5 triplet regimens, were most sensitive to changes in the cost of treatment (i.e., drug acquisition costs; see Appendix, available in online article). A 20\% increase in drug acquisition costs led to expected monthly costs of $\$ 16,393$ (DARA/BOR/DEX), \$26,535 (IXA/LEN/DEX), \$27,007 (ELO/ LEN/DEX), \$31,501 (DARA/LEN/DEX), and \$32,618 (CAR/ LEN/DEX). Following drug acquisition costs, all regimens were also sensitive to the modeled treatment duration, although to a lesser degree than the cost of therapy. Beyond treatment duration and acquisition costs, the cost of subsequent therapy and $\mathrm{AE}$ management were the next largest drivers of cost.

A summary of the scenario analyses designed to test the impact of plausible alternative model inputs for each triplet regimen are presented in Table 4. For all regimens, setting the 1-year PFS rate equal to $100 \%$ yielded the greatest monthly cost; those costs were $\$ 16,773$ (DARA/BOR/DEX), $\$ 26,112$ (IXA/LEN/DEX), \$28,423 (ELO/LEN/DEX), \$28,536 (DARA/ LEN/DEX), and $\$ 30,657$ (CAR/LEN/DEX). The effect of PFS rates of $75 \%$ and $50 \%$ for each triplet therapy are also presented in Table 4. The greatest monthly costs for each regimen, excluding the cost of a full year of treatment as previously calculated, was $\$ 15,630$ (DARA/BOR/DEX), $\$ 24,177$ (IXA/LEN/ DEX), \$26,319 (ELO/LEN/DEX), \$27,305 (DARA/LEN/DEX), and $\$ 28,351$ (CAR/LEN/DEX).

\section{Discussion}

The efficacy of triplet regimens for the treatment of patients with relapsed or refractory MM has been established in randomized controlled trials, approved by the FDA, and recommended by the NCCN guidelines as the preferred care for previously treated MM patients. ${ }^{4,6}$ With limited time on market since FDA approval, published real-world estimates of the cost of these therapies have been limited by the lack of mature claims data that capture use of triplet therapies. ${ }^{7,8}$ With the increasing focus on value-based care, decision makers are 
TABLE 3 Estimated Treatment-Related Costs of Each Triplet and Contribution by Key Cost Categories

\begin{tabular}{|c|c|c|c|c|c|}
\hline & $\begin{array}{c}\text { DARA/BOR/DEX } \\
\$\end{array}$ & $\begin{array}{c}\text { DARA/LEN/DEX } \\
\$\end{array}$ & $\begin{array}{c}\text { CAR/LEN/DEX } \\
\$\end{array}$ & $\begin{array}{c}\text { IXA/LEN/DEX } \\
\$\end{array}$ & $\begin{array}{c}\text { ELO/LEN/DEX } \\
\$\end{array}$ \\
\hline Total cost per patient in year 1 & $166,675.84$ & $316,918.87$ & $329,178.01$ & $266,769.45$ & $291,864.53$ \\
\hline Average monthly cost per patient ${ }^{\mathrm{a}}$ & $13,889.65$ & $26,409.91$ & $27,431.50$ & $22,230.79$ & $24,322.04$ \\
\hline \multicolumn{6}{|c|}{ Average monthly cost per patient in year 1 , by cost category $b$} \\
\hline Drug acquisition costs & $12,515.87$ & $25,453.43$ & $25,931.85$ & $21,518.76$ & $22,982.02$ \\
\hline Administration costs & 489.45 & 403.53 & 808.86 & 0.00 & 375.41 \\
\hline Monitoring & 82.49 & 83.11 & 130.10 & 139.01 & 76.33 \\
\hline Comedications and l-time costs & 3.64 & 2.36 & 19.75 & 4.83 & 27.77 \\
\hline $\mathrm{AE}$ costs & 159.28 & 214.32 & 186.83 & 60.37 & 367.01 \\
\hline Subsequent therapy costs & 638.92 & 253.16 & 354.12 & 507.82 & 493.51 \\
\hline \multicolumn{6}{|c|}{$\begin{array}{l}\text { Dollar values shown are rounded to } 2 \text { significant digits. Model calculations were performed without rounding. } \\
\text { The average monthly cost of each cost component was calculated by multiplying the percent contribution of each cost category over } 1 \text { year by the average monthly cost per } \\
\text { patient. Therefore, the presented average monthly costs represent the average cost per month over a 1-year time period and are not representative of actual costs incurred in } \\
\text { any } 1 \text { month during that year. } \\
\text { AE = adverse event; } B O R=\text { bortezomib; CAR=carfilzomib; DARA=daratumumab; DEX=dexamethasone; ELO=elotuzumab; IXA=ixazomib; LEN=lenalidomide. }\end{array}$} \\
\hline
\end{tabular}

more frequently turning to economic analyses to understand the value of therapies. ${ }^{11}$ This study sought to inform PBMs and other U.S. health care decision makers by estimating the average per-patient cost of FDA-approved and NCCN-recommended (preferred, category 1) triplet therapies for patients with previously treated MM. Through the direct comparison of the cost of therapy with each triplet regimen and the absence of market share assumptions, this analysis avoids limitations associated with estimating the market utilization of each treatment.

This analysis accounted for many direct costs associated with the treatment of relapsed or refractory MM patients over a 1-year time horizon, but because our analyses omitted indirect costs of care and focused only on MM treatment costs, the total cost of care for all MM patients was not calculated. The average cost per patient was calculated by applying the triplet and subsequent therapy costs to the probability of remaining on treatment/progression at 12 months (estimated from the modeled PFS curves). The model estimated the average monthly cost of treating relapsed or refractory MM with DARA/BOR/ DEX, IXA/LEN/DEX, ELO/LEN/DEX, DARA/LEN/DEX, and CAR/LEN/DEX to be $\$ 13,890, \$ 22,231, \$ 24,322, \$ 26,410$ and $\$ 27,432$, respectively. DARA/BOR/DEX was found to be the least costly triplet therapy, which was likely due to the cessation of BOR dosing after week 24 per the CASTOR trial and FDA-approved label, the lowest modeled treatment duration of the 5 triplets tested, and the reduced frequency of administration of the remaining drug components (i.e., DARA) over time. To our knowledge, this represents the first study to directly compare the expected cost of treating patients with previously treated MM for 1 year with the 5 NCCN-recommended and preferred triplet regimens.

Sensitivity analyses revealed that these findings were most dependent on the cost of drug acquisition and the modeled treatment duration. Drug acquisition costs within the model were based on WAC ${ }^{18}$ rather than ASP because some therapies continue not to be equivalently listed by Medicare. ${ }^{30}$ Scenario analyses found that the average monthly cost of each regimen varied less than $5 \%$ when the model was parameterized with ASP rather than WAC, indicating that the difference between WAC and ASP had a small effect on model results.

Scenario analyses to assess uncertainty in the modeled treatment duration were performed by testing alternative assumptions for each triplet, including (a) the next best fit curve, (b) a Weibull distribution, (c) equivalent efficacy, and (d) a full year of treatment. While these scenario analyses affected the estimated cost per month of each triplet regimen, they did not change the order of least to most expensive triplet therapy (DARA/BOR/DEX $<$ IXA/LEN/DEX $<$ ELO/LEN/DEX $<$ DARA/ LEN/DEX $<$ CAR/LEN/DEX). Scenario analyses to test the cost of treating patients with different weights ( $76.5 \mathrm{~kg}$ and $85.5 \mathrm{~kg}$ ) and surface areas $\left(1.68 \mathrm{~m}^{2}\right.$ and $\left.2.0 \mathrm{~m}^{2}\right)$ also failed to alter the order of triplet therapies; the tested values were representative of variation in the data reported in the publications from which weight and body surface area were derived. . $^{2,20}$

Although all regimens are sensitive to vial wastage, ELO/ LEN/DEX was particularly dependent on correct vial size selection. For example, treating a patient who required three $300 \mathrm{mg}$ vials (ELO is available in $300 \mathrm{mg}$ and $400 \mathrm{mg}$ units) resulted in an average monthly cost of $\$ 25,610$, while treatment with three $400 \mathrm{mg}$ vials resulted in an average monthly cost of $\$ 29,500$, which would make ELO/LEN/DEX the second most expensive triplet.

The scenario and sensitivity analyses provide a plausible range of per-patient costs for these $5 \mathrm{MM}$ triplets, with the scenario assuming 100\% PFS representing a plausible maximum cost per patient for each triplet; the real cost may fall between these estimates and likely closer to the base-case estimate. 


\section{A U.S. Cost Analysis of Triplet Regimens for Patients with Previously Treated Multiple Myeloma}

\section{TABLE 4 Scenario Analyses to Test Plausible Alternative Model Inputs}

\begin{tabular}{|c|c|c|c|c|c|c|c|c|}
\hline \multirow[b]{2}{*}{ Inputs } & \multirow{2}{*}{$\begin{array}{l}\text { Original } \\
\text { Value }\end{array}$} & \multicolumn{2}{|c|}{ Scenario Values } & \multirow[b]{2}{*}{ DVd, $\$$ a } & \multirow[b]{2}{*}{ DRd, $\$$ a } & \multirow[b]{2}{*}{$\mathrm{KRd}, \$ \mathrm{a}$} & \multirow[b]{2}{*}{ IRd, \$a } & \multirow[b]{2}{*}{ ERd, $\$ \mathrm{a}$} \\
\hline & & Low & High & & & & & \\
\hline Original value & & & & $13,889.65$ & $26,409.91$ & $27,431.50$ & $22,230.79$ & $24,322.04$ \\
\hline \multicolumn{9}{|c|}{ Unit cost parameters updated with ASP rather than WAC } \\
\hline DARA & $\$ 50.98$ & $\$ 50.39$ & & $13,783.88$ & $26,275.25$ & & & \\
\hline ELO & $\$ 6.19$ & & $\$ 6.35$ & & & & & $24,583.96$ \\
\hline CAR & $\$ 37.57$ & $\$ 34.11$ & & & & $26,271.75$ & & \\
\hline $\mathrm{BOR}$ & $\$ 45.80$ & & $\$ 46.66$ & $13,953.12$ & & & & \\
\hline \multicolumn{9}{|c|}{ Range of BSA and weight ${ }^{c}$} \\
\hline $\mathrm{BSA}$ & $1.86 \mathrm{~m}^{2}$ & $1.68 \mathrm{~m}^{2}$ & $2.00 \mathrm{~m}^{2}$ & NC & NC & NC & NC & NC \\
\hline Weight & $79.4 \mathrm{~kg}$ & $76.5 \mathrm{~kg}$ & & NC & NC & NC & NC & NC \\
\hline Weight & $79.4 \mathrm{~kg}$ & & $85.5 \mathrm{~kg}$ & $14,592.72$ & $27,305.01$ & NC & NC & $25,609.68$ \\
\hline \multicolumn{9}{|c|}{ Management cost of infusion reactions } \\
\hline IR cost & $\$ 13,300.50$ & $\$ 9,015.54$ & & $13,857.52$ & $26,392.05$ & NC & NC & NC \\
\hline IR cost & $\$ 13,300.50$ & & $\$ 19,901.65$ & $13,939.16$ & $26,437.41$ & NC & NC & NC \\
\hline \multicolumn{9}{|c|}{ Treatment duration ${ }^{\mathrm{d}}-$ next best curve fit $^{\mathrm{e}}$} \\
\hline DVd & $57.6 \%$ & & $59.1 \%$ & $13,991.64$ & & & & \\
\hline$\overline{\mathrm{DRd}}$ & $83.2 \%$ & & $83.3 \%$ & & $26,422.56$ & & & \\
\hline KRd & $76.5 \%$ & & $76.5 \%$ & & & NC & & \\
\hline IRd & $66.3 \%$ & & $66.5 \%$ & & & & $22,253.82$ & \\
\hline ERd & $67.2 \%$ & $66.8 \%$ & & & & & & $24,265.74$ \\
\hline \multicolumn{9}{|c|}{ Treatment duration-all Weibull } \\
\hline DVd & $57.6 \%$ & & $57.9 \%$ & $13,910.05$ & & & & \\
\hline$\overline{\mathrm{DRd}}$ & $83.2 \%$ & & $83.4 \%$ & & $26,435.21$ & & & \\
\hline KRd & $76.5 \%$ & Same as above & & & & & & \\
\hline IRd & $66.3 \%$ & & $66.9 \%$ & & & & $22,299.89$ & \\
\hline ERd & $67.2 \%$ & & $67.7 \%$ & & & & & $24,378.43$ \\
\hline \multicolumn{9}{|c|}{ Treatment duration-all equivalent } \\
\hline DVd & $57.6 \%$ & $57.6 \%$ & & $\mathrm{NC}$ & & & & \\
\hline $\mathrm{DVd}$ & $57.6 \%$ & & $83.2 \%$ & $15,630.31$ & & & & \\
\hline DRd & $83.2 \%$ & $57.6 \%$ & & & $23,170.62$ & & & \\
\hline$\overline{\mathrm{DRd}}$ & $83.2 \%$ & & $83.2 \%$ & & NC & & & \\
\hline KRd & $76.5 \%$ & $57.6 \%$ & & & & $24,837.31$ & & \\
\hline KRd & $76.5 \%$ & & $83.2 \%$ & & & $28,351.14$ & & \\
\hline IRd & $66.3 \%$ & $57.6 \%$ & & & & & $21,228.85$ & \\
\hline IRd & $66.3 \%$ & & $83.2 \%$ & & & & $24,177.08$ & \\
\hline ERd & $67.2 \%$ & $57.6 \%$ & & & & & & $23,113.82$ \\
\hline ERd & $67.2 \%$ & & $83.2 \%$ & & & & & $26,319.18$ \\
\hline \multicolumn{9}{|c|}{ Treatment duration-triplet therapy for 6,9 , or 12 months } \\
\hline \multirow{3}{*}{ All regimens } & \multirow{3}{*}{ Various } & 12 month & s (100\%) & $16,772.61$ & $28,535.69$ & $30,657.09$ & $26,111.85$ & $28,422.71$ \\
\hline & & 9 month & $\mathrm{s}(75 \%)$ & $15,072.75$ & $25,372.32$ & $27,225.61$ & $23,232.72$ & $25,292.46$ \\
\hline & & 6 month & s (50\%) & $13,372.90$ & $22,208.96$ & $23,794.14$ & $20,353.60$ & $22,162.22$ \\
\hline \multicolumn{9}{|c|}{ Impact of Survival-No Subsequent Therapy Costs } \\
\hline All regimens & Various & $\begin{array}{l}\text { No postprogre } \\
\text { costs (i.e., as } \\
\text { mortality rate }\end{array}$ & $\begin{array}{l}\text { ssion therapy } \\
\text { sumed } 100 \% \\
\text { n progression) }\end{array}$ & $13,250.74$ & $26,156.75$ & $27,077.38$ & $21,722.97$ & $23,828.54$ \\
\hline
\end{tabular}

a Model inputs and results of the scenario analyses are presented as the average monthly cost (2018 USD) in the first year of treatment.

${ }^{b}$ ASP as reported by Medicare, is shown here. In the model, Medicare reported that ASP was used to estimate the cost of commercially available unit sizes.

'The high and low BSA were taken from the interquartile range reported by Baker et al. (2002), ${ }^{19}$ and the range for the weight was taken from Talamo et al. (2013).20

dTreatment duration refers to the estimated proportion of patients remaining on therapy at 12 months.

'The next best curve fit for DVd, DRd, KRd, IRd, and ERd were log-normal, Gompertz, Weibull, log-logistic, and log-normal, respectively.

$A S P=$ average selling price; $B O R=$ bortezomib; $B S A=$ body surface area; $C A R=$ carfilzomib; $D A R A=$ daratumumab; DRd =DARA/LEN/DEX; DVd=DARA $/ B O R / D E X$;

$D E X=$ dexamethasone $; E L O=$ elotuzumab; ERd = ELO/LEN/DEX; IRd =IXA/LEN/DEX; IR=infusion reactions; IXA=ixazomib; KRd=CAR/LEN/DEX; LEN=lenalidomide; $N C=$ no change; $U S D=$ U.S. dollars; $W A C=$ wholesale acquisition cost. 
The cost of administration was highest for CAR/LEN/DEX, as a result of this regimen requiring 6 infusions per cycle from cycle 2 until cycle 13 (i.e., the first 11 months of treatment; Table 1$){ }^{16}$ while other regimens decreased infusions to 2 or less per cycle by cycle 4 (i.e., within 2-3 months of treatment initiation). ${ }^{14,15,17}$ Recurring and 1-time monitoring costs were fairly consistent across the regimens and represented a minor contribution to total costs.

The cost of AE management was also relatively low, comprising $1.5 \%$ or less of the expected monthly expenditure for all regimens. Some uncertainty exists around the costing of AE management because, to our knowledge, no study has performed a rigorous claims-based assessment of the costs associated with AEs induced by MM treatments. However, as $\mathrm{AE}$ management costs were derived from a previously published MM economic model, ${ }^{26}$ it was not expected that alternative inputs for the cost of AE management would significantly change model results.

This study was a cost analysis, and while the methodological approach differed from a standard budget impact analysis or cost-effectiveness analysis, there are some shared elements, such as a dependence on the expected duration of therapy and the objective of quantifying the cost of therapy over a given time horizon. The similarities across economic analyses enable comparisons with the published literature. Roy et al. generated an economic model to estimate the cost of singlet, doublet, and triplet therapies for patients with relapsed or refractory MM. ${ }^{26}$ CAR/LEN/DEX was the only triplet examined by the current study and Roy et al., which estimated the monthly cost (2015 U.S. dollars) of CAR/LEN/DEX to be $\$ 24,293$ and $\$ 27,422$, to a Medicare and U.S. commercial plan, respectively. ${ }^{26}$ In the current study, treatment with CAR/LEN/DEX was estimated to cost $\$ 27,432$ per month, which is in line with Roy et al. Further, Roy et al. reported the cost of triplet therapies to vary between $\$ 17,055$ and $\$ 27,422$ per month ${ }^{26}$; this range is consistent with the findings of the current study, with the exception of DARA/ BOR/DEX, which was estimated to cost $\$ 13,890$ per month. In contrast, a recent claims-based estimate of the monthly cost of treating relapsed or refractory MM found that the cost varied between $\$ 35,266$ and $\$ 47,417 .{ }^{8}$ Real-world evidence comparing health care utilization and pharmacy costs after the introduction of triplet therapies and increased competition in the field will be valuable to future studies.

The cost of the CAR/LEN/DEX triplet has also been assessed in 2 other economic models. Jakubowiak et al. (2016) estimated the cost of CAR/LEN/DEX therapy to be $\$ 363,432$ over a median 3.79 progression-free life-years, ${ }^{31}$ while our estimate of only the first year of therapy was $\$ 329,178$. The relatively minor difference between the estimated cost of 1 year and 3.79 years of CAR/LEN/DEX treatment is likely due to the dosing schedule, since CAR administration stops after cycle
18 ( 1.4 years; Table 1), leading to a marked decrease in cost accumulation after that point. In the cost-effectiveness analysis and budget impact model reported by Bloudek et al. (2016), the yearly cost of CAR/LEN/DEX was found to be approximately $\$ 231,124-\$ 232,164 .^{32}$ These results are similar to ours, since the annual cost reported by Bloudek et al. did not consider subsequent therapy costs. Finally, Carlson et al. (2018) examined the cost-effectiveness of treatment with CAR, ELO, IXA, DARA, and panobinostat (PANO) in combination with LEN or BOR plus DEX for patients with relapsed or refractory MM over a lifetime time horizon using a partition survival model. ${ }^{33}$ DARA/BOR/DEX and PANO/BOR/DEX were found to be the most cost-effective options, with DARA regimens estimated to offer the greatest increase in life-years and quality-adjusted lifeyears. ${ }^{33}$ The analysis presented in this study differs from the work of Carlson et al. because this study is a practical cost comparison designed to help PBMs and other U.S. health care decision makers to understand the expected initial cost of treating patients with relapsed or refractory MM. Furthermore, while Carlson et al. completed a rigorous cost-effectiveness analysis, partitioned survival models are reliant on the extrapolation of survival data to estimate costs and survival; in contrast, the current analysis was completed without extrapolation of survival curves and associated assumptions.

Other available economic analyses for MM treatments have evaluated the cost-effectiveness of monotherapies and doublets, such as CAR/DEX and BOR/DEX, ${ }^{34}$ POM/DEX, CAR monotherapy, and DARA monotherapy, ${ }^{35}$ or PANO/DEX therapy. ${ }^{32}$ These analyses estimate the cost of treating a relapsed or refractory MM patient with monotherapies or doublets to be between $\$ 73,272-\$ 178,967$ annually. ${ }^{32,34,35}$ In addition, a recent claimsbased assessment of the cost of treating 2,551 Medicare beneficiaries with non-chemotherapeutic agents between 2000 and 2009 determined the average 12 -month cost to be $\$ 144,665 .^{36}$ Given the time frame of that study, the agents captured would not have been focused on triplet therapies and therefore would not be comparable to this study.

As real-world drug and health care utilization rates become available for triplet therapies, a better understanding of the actual average annual cost of triplet therapy to U.S. health care plans will emerge. This cost analysis, however, has estimated the cost of treating a single relapsed or refractory MM patient with 5 triplet regimens over a 1-year time horizon after treatment initiation. For U.S. payers, these per-patient costs can be used alongside treatment utilization estimates to determine total costs to their plans.

\section{Limitations}

Although common in economic modeling, some data inputs required assumptions that may limit interpretation of the results. Therefore, the estimated 1-year and average monthly 
treatment-related costs of triplet regimens used in patients with relapsed or refractory MM may not be generalizable to all U.S. health plans.

The objective of this analysis was to assess the cost of drug therapy for an average patient with relapsed or refractory MM. This analysis did not account for additional costs required to manage patient subpopulations because the number of MM patients at risk were not consistently reported for all treatments. For example, prophylactic care may depend on variable patient characteristics, hospital protocols, and clinician discretion. Further, given that prophylaxis may involve relatively inexpensive care, such as increased comedications or monitoring, it is unlikely that this would markedly affect the estimated cost per patient. Moreover, core-regimen dose-reductions stemming from the management of AEs or at-risk/elderly patients were not considered. Dose reductions would lead to reduced drug acquisition costs.

To estimate the average duration of treatment with each triplet regimen within the model, PFS Kaplan-Meier data were modeled and extrapolated. Extrapolation of PFS Kaplan-Meier data were required for this analysis because, at the time of model building, median PFS had not yet been reached in the CASTOR or POLLUX trials of DARA/BOR/DEX and DARA/ LEN/DEX, respectively. ${ }^{5,37}$ The decision to use modeled efficacy data remains valid, as POLLUX has still not yet reached median PFS. ${ }^{38}$ To control for the use of this approach, the same methodology for curve extrapolation was applied to all triplet regimens, and scenario analyses were performed to examine alternative plausible data inputs. Nonetheless, this approach did not allow for the use of indirect treatment comparisons nor correction of data based on differences in trial populations. As such, decision makers are encouraged to consider results presented here in the context of the performance of triplets for patients within their health plans.

To our knowledge, a U.S. claims-based assessment of AE management costs in relapsed or refractory MM patients has not been completed. Thus, the cost of managing AEs in relapsed or refractory MM patients was primarily derived from the economic analysis performed by Roy et al. in $2015,{ }^{26}$ which used AE costs from patients with a mix of cancer types. It was assumed that the cost of managing AEs arising from chemotherapy or triplet therapy would be similar because management costs for AEs with similar clinical presentation should be treated similarly. Assumptions were used to estimate the cost of managing AEs not reported by Roy et al. Because the incremental difference between the cost of managing AEs with similar presentation should be minor, it is unlikely that these assumptions significantly affected the results of this analysis. This analysis considered AEs that were reported within the FDA prescribing information of each therapy. Since FDA prescribing information for these therapies presents the number of patients experiencing an $\mathrm{AE}$, rather than the total number of events, it is possible that the presented analyses underestimated the cost of AE management due to not accounting for recurrent AEs.

The cost of managing infusion reactions available in the literature was not specific to any of the included treatments ${ }^{27}$; however, uncertainty introduced by this approach would affect all comparators proportionate to the rate of infusion reactions. Because only DARA-based triplet therapies reported a rate of infusion reactions that met the inclusion criteria of this study, and sensitivity analyses were performed to examine the effect of all infusion reactions being managed in the inpatient or outpatient setting, this assumption was deemed acceptable, since the actual cost is likely to be within this range. Moreover, scenario analyses examining the cost of managing infusion reactions were performed and found to have had a minor effect on model results.

\section{Conclusions}

This cost analysis can be used to help inform decision makers and payers regarding the cost of triplet regimens for the treatment of relapsed or refractory MM. The analysis suggests that 1 year after treatment initiation, DARA/BOR/DEX may have the lowest cost per treated patient, while CAR/LEN/DEX therapy may have the highest cost per treated patient. Since these are modeled costs, data assessing real-world health care resource and treatment utilization would facilitate a better understanding of the real-world costs of triplet therapies in the United States.

\section{Authors}

SARAH HOLLMANN, MBiotech; DANIEL MOLDAVER, PhD; NIK GOYERT, MA; and DANIEL GRIMA, MSc, Cornerstone Research Group, Burlington, Ontario, Canada. ERIC M. MAIESE, MHS, PhD, Janssen Scientific Affairs, Horsham, Pennsylvania.

AUTHOR CORRESPONDENCE: Eric M. Maiese, MHS, PhD, Director, HEOR, Janssen Scientific Affairs, 800 Ridgeview Dr., Horsham, PA 19044. Tel.: 215.356.1966; E-mail: emaiese@its.jnj.com.

\section{DISCLOSURES}

This study was funded by Janssen Scientific Affairs, which employs Maiese and funded Cornerstone Research Group, a health economic consulting group, to complete the cost analysis, interpret data, and develop the manuscript. Janssen was involved in the design of the analysis, interpretation of results, and manuscript development and approval. Grima is a founding partner of Cornerstone Research Group, which employs Hollmann, Goyert, and Moldaver. Hollmann, Goyert, and Moldaver were responsible for creation of the economic model.

This work was peer-reviewed and presented as an abstract at the Lymphoma and Myeloma 2017 International Congress; October 26-28, 2017; New York, NY. 


\section{REFERENCES}

1. Siegel RL, Miller KD, Jemal A. Cancer statistics, 2016. CA Cancer J Clin. 2016;66(1):7-30.

2. National Cancer Institute, SEER Surveillance Epidemiology and End Results Program. SEER stat fact sheets: myeloma. Available at: https://seer. cancer.gov/statfacts/html/mulmy.html. Accessed March 8, 2019.

3. Kumar SK, Lee JH, Lahuerta JJ, et al. Risk of progression and survival in multiple myeloma relapsing after therapy with IMiDs and bortezomib: a multicenter international myeloma working group study. Leukemia. 2012;26(1):149-57.

4. Rajkumar SV, Kumar S. Multiple myeloma: diagnosis and treatment. Mayo Clin Proc. 2016;91(1):101-19.

5. Dimopoulos MA, Oriol A, Nahi H, et al. Daratumumab, lenalidomide, and dexamethasone for multiple myeloma. N Engl J Med. 2016;375(14):1319-31.

6. National Comprehensive Cancer Network. Clincal practice guidelines in oncology: multiple myeloma. Version 2.2017. Available at: http://www.nccn. org/professionals/physician_gls/f_guidelines.asp. Accessed March 8, 2019.

7. Arikian SR, Milentijevic D, Binder G, et al. Patterns of total cost and economic consequences of progression for patients with newly diagnosed multiple myeloma. Curr Med Res Opin. 2015;31(6):1105-15.

8. MacEwan JP, Batt K, Yin W, et al. Economic burden of multiple myeloma among patients in successive lines of therapy in the United States. Leuk Lymphoma. 2018;59(4):941-49.

9. Teitelbaum A, Ba-Mancini A, Huang H, Henk HJ. Health care costs and resource utilization, including patient burden, associated with novel-agentbased treatment versus other therapies for multiple myeloma: findings using real-world claims data. Oncologist. 2013;18(1):37-45.

10. Keehan SP, Cuckler GA, Sisko AM, et al. National health expenditure projections, 2014-24: spending growth faster than recent trends. Health Aff (Millwood). 2015;34(8):1407-17.

11. Sorenson C, Lavezzari G, Daniel G, et al. Advancing value assessment in the United States: a multistakeholder perspective. Value Health. 2017;20(2):299-307.

12. Hollmann S, Goyert N, Moldaver D, Grima D, Maiese E. U.S. budget impact analysis for daratumumab in combination with lenalidomide and dexamethasone, bortezomib and dexamethasone, or pomalidomide and dexamethasone for the treatment of patients with relapsed/refractory multiple myeloma [abstract]. J Manag Care Spec Pharm. 2017;23(10-a):S32. Available at: https://www.jmcp.org/doi/10.18553/jmcp.2017.23.10-a.sl.

13. Sullivan SD, Mauskopf JA, Augustovski F, et al. Budget impact analysis-principles of good practice: report of the ISPOR 2012 Budget Impact Analysis Good Practice II Task Force. Value Health. 2014;17(1):5-14.

14. DARZALEX (daratumumab) injection, for intravenous use. Janssen. Revised June 2017. Available at: https://www.accessdata.fda.gov/drugsatfda_ docs/label/2017/761036s005lbl.pdf. Accessed March 10, 2019.

15. EMPLICITI (elotuzumab) for injection, for intravenous use. Bristol-Myers Squibb. Revised November 2015. Available at: https://www.accessdata.fda.gov/ drugsatfda_docs/label/2015/761035s000lbl.pdf. Accessed March 10, 2019.

16. KYPROLIS (carfilzomib) for injection, for intravenous use. Onyx Pharmaceuticals.Revised August 2016. Available at: http://www.accessdata.fda.gov/ drugsatfda_docs/label/2016/202714s013lbl.pdf. Accessed March 10, 2019.

17. NINLARO (ixazomib) capsules, for oral use. Millennium Pharmaceuticals. Revised November 2015. Available at http://www.accessdata.fda.gov/drugsatfda_docs/label/2015/208462lbl.pdf. Accessed March 10, 2019.

18. Truven Health Analytics, IBM Watson Health. IBM Micromedes RED BOOK Online. Database. Retrieved May 22, 2018. Available at: https:// www.ibm.com/us-en/marketplace/micromedex-red-book?cm_mc_ uid $=15468529316115235010397 \&$ cm_mc_sid_50200000 $=67289411552$ 260634053\&cm_mc_sid_52640000=47897251552260634090. Accessed March 10, 2019.
19. Baker SD, Verweij J, Rowinsky EK, et al. Role of body surface area in dosing of investigational anticancer agents in adults, 1991-2001. J Natl Cancer Inst. 2002;94(24):1883-88.

20. Talamo G, Zhou J, Dolloff NG, et al. Retrospective study of body weight in patients with multiple myeloma through different stages of the disease. Metab Nutr Oncol. 2013;01(01):e7-ell. Available at: https://www.thiemeconnect.de/products/ejournals/html/10.1055/s-0033-1358760. Accessed March 10, 2019

21. Centers for Medicare \& Medicaid Services. 2018 Clinical Diagnostic Laboratory Fee Schedule. Available at: https://www.cms.gov/Medicare/ Medicare-Fee-for-Service-Payment/ClinicalLabFeeSched/ClinicalLaboratory-Fee-Schedule-Files-Items/18CLAB.html?DLPage $=1 \& D L E n t r i e s=1$ 0\&DLSort=2\&DLSortDir=descending. Accessed March 10, 2019.

22. Centers for Medicare \& Medicaid Services. Physician Fee Schedule search. Available at: https://www.cms.gov/apps/physician-fee-schedule/ search/search-criteria.aspx. Accessed March 10, 2019.

23. VELCADE (bortezomib) for injection, for subcutaneous or intravenous use. Millennium Pharmaceuticals. Revised September 2015. Available at: http:// www.accessdata.fda.gov/drugsatfda_docs/label/2015/021602s042lbl.pdf. Accessed March 10, 2019.

24. Ollendorf D, Chapman R, Khan S, et al. Treatment options for relapsed or refractory multiple myeloma: effectiveness, value, and value-based price benchmarks. Evidence report. Institute for Clinical and Economic Review. May 5, 2016. Available at: https://icer-review.org/wp-content/ uploads/2016/05/MWCEPAC_MM_Evidence_Report_050516-002.pdf. Accessed March 10, 2019.

25. Farr A, Stott-Miller M, Varker H, Spencer D, Shah M, Chen C. Treatment sequencing patterns associated with elderly patients with relapsed/ refractory multiple myeloma (MM) in the U.S. community setting. Blood. 2015;126(23):5392.

26. Roy A, Kish JK, Bloudek L, et al. Estimating the costs of therapy in patients with relapsed and/or refractory multiple myeloma: a model framework. Am Health Drug Benefits. 2015;8(4):204-15.

27. Foley KA, Wang PF, Barber BL, et al. Clinical and economic impact of infusion reactions in patients with colorectal cancer treated with cetuximab. Ann Oncol. 2010;21(7):1455-61.

28. Hollander-Rodriguez JC, Calvert JF Jr. Hyperkalemia. Am Fam Physician. 2006;73(2):283-90.

29. Jordan M, Caesar J. Hypokalaemia: improving the investigation, management and therapeutic monitoring of hypokalaemic medical inpatients at a district general hospital. BMJ Qual Improv Rep. 2015;4(1):u209049.w203670. Available at: https://www.ncbi.nlm.nih.gov/pmc/articles/PMC4693097/. Accessed March 10, 2019.

30. Centers for Medicare \& Medicaid Services. April 2018 ASP pricing file. Effective April 1, 2018 through June 30, 2018. Available at: https://www.cms. gov/Medicare/Medicare-Fee-for-Service-Part-B-Drugs/McrPartBDrugAvgSalesPri ce/2018ASPFiles.html. Accessed March 10, 2019.

31. Jakubowiak AJ, Campioni M, Benedict A, et al. Cost-effectiveness of adding carfilzomib to lenalidomide and dexamethasone in relapsed multiple myeloma from a U.S. perspective. J Med Econ. 2016;19(11):1061-74.

32. Bloudek L, Roy A, Kish JK, et al. Estimating the economic impact of adding panobinostat to a U.S. formulary for relapsed and/or refractory multiple myeloma: a budget impact and cost-benefit model. J Manag Care Spec Pharm. 2016;22(8):991-1002. Available at: https://www.jmcp.org/doi/10.18553/ jmcp.2016.22.8.991.

33. Carlson JJ, Guzauskas GF, Chapman RH, et al. Cost-effectiveness of drugs to treat relapsed/refractory multiple myeloma in the United States. J Manag Care Spec Pharm. 2018;24(1):29-38. Available at: https://www.jmcp. org/doi/10.18553/jmcp.2018.24.1.29. 
34. Jakubowiak AJ, Houisse I, Majer I, et al. Cost-effectiveness of carfilzomib plus dexamethasone compared with bortezomib plus dexamethasone for patients with relapsed or refractory multiple myeloma in the United States. Expert Rev Hematol. 2017;10(12):1107-19.

35. Pelligra CG, Parikh K, Guo S, et al. Cost-effectiveness of pomalidomide, carfilzomib, and daratumumab for the treatment of patients with heavily pretreated relapsed-refractory multiple myeloma in the United States. Clin Ther. 2017;39(10):1986-2005.e5.

36. Chen Y, Lairson DR, Chan W, Huo J, Du XL. Cost-effectiveness of novel agents in Medicare patients with multiple myeloma: findings from a U.S.

payer's perspective. J Manag Care Spec Pharm. 2017;23(8):831-43. Available at: https://www.jmcp.org/doi/10.18553/jmcp.2017.23.8.831.

37. Palumbo A, Chanan-Khan A, Weisel K, et al. Daratumumab, bortezomib, and dexamethasone for multiple myeloma. N Engl J Med. 2016;375(8):754-66.
38. Weisel KC, San Miguel J, Cook G, et al. Efficacy of daratumumab in combination with lenalidomide plus dexamethasone (DRd) or bortezomib plus dexamethasone (DVd) in relapsed or refractory multiple myeloma (RRMM) based on cytogenetic risk status. J Clin Oncol. 2017;35(15 Suppl): Abstract 8006. Available at: http://ascopubs.org/doi/abs/10.1200/JCO. 2017.35.15_suppl.8006. Accessed March 10, 2019.

39. Stewart AK, Rajkumar SV, Dimopoulos MA, et al. Carfilzomib, lenalidomide, and dexamethasone for relapsed multiple myeloma. N Engl J Med. 2015;372(2):142-52.

40. Moreau P, Masszi T, Grzasko N, et al. Oral ixazomib, lenalidomide, and dexamethasone for multiple myeloma. N Engl J Med. 2016;374(17):1621-34.

41. Lonial S, Dimopoulos M, Palumbo A, et al. Elotuzumab therapy for relapsed or refractory multiple myeloma. New Engl J Med. 2015;373(7):621-31.

\section{APPENDIX Tornado Diagram Depicting Results of the One-Way Sensitivity Analysis}

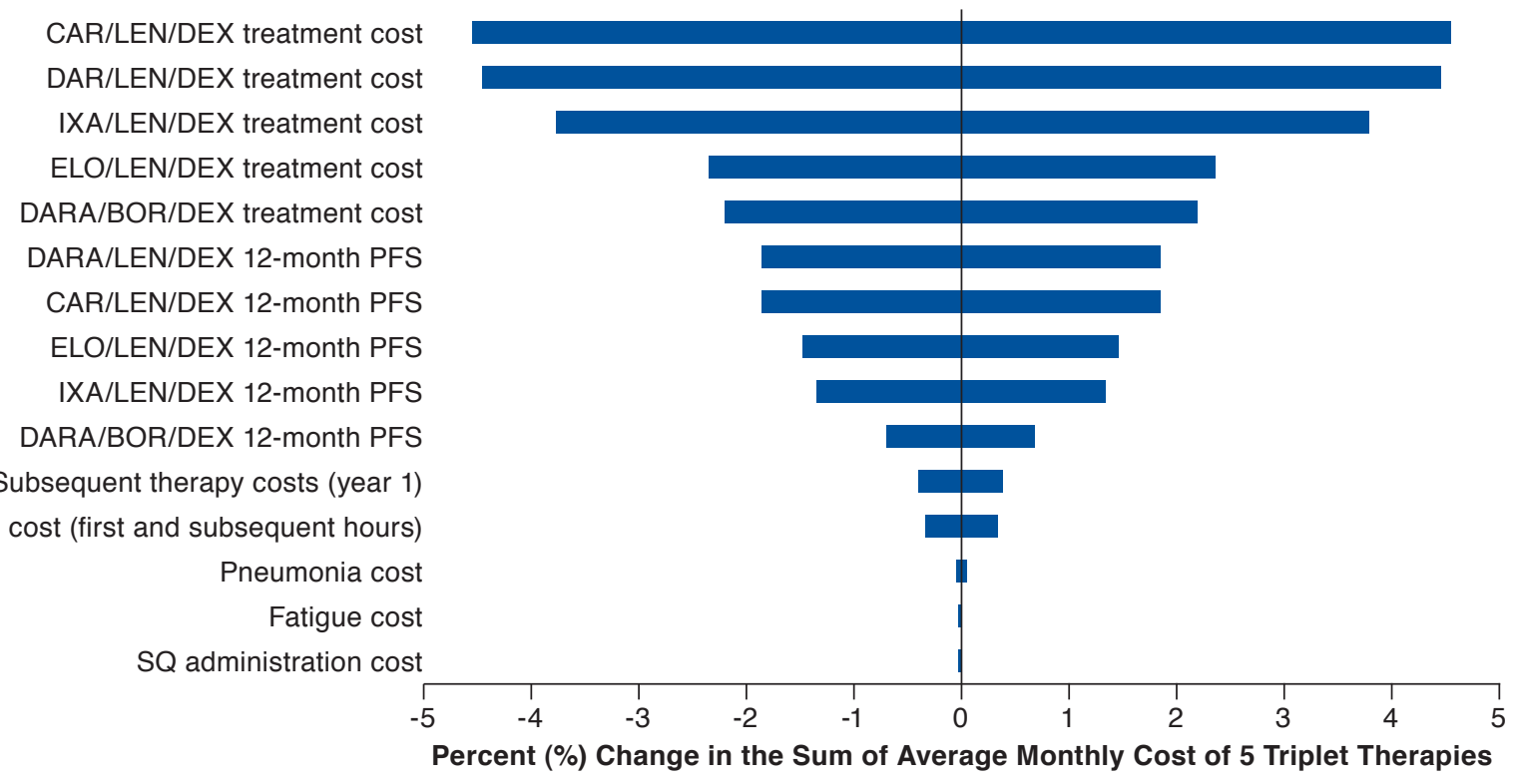

BOR=bortezomib; $C A R=$ carfilzomib; DARA =daratumumab; DEX=dexamethasone; ELO=elotuzumab; IV=intravenous; IXA=ixazomib; LEN =lenalidomide; PFS = progression-free survival; $S Q=$ subcutaneous. 